\title{
VALORES NA EDUCAÇÃO EM SAÚDE E A FORMAÇÃO PROFISSIONAL
}

\author{
VALUES IN HEALTH EDUCATION AND VOCATIONAL TRAINING
}

\author{
Amâncio António de Sousa Carvalho ${ }^{1}$ \\ Graça Simões de Carvalho \\ Vítor Manuel Costa Pereira Rodrigues ${ }^{3}$
}

Resumo O presente estudo traçou, como objetivo geral, analisar o efeito da formação na aquisição de valores em educação em saúde dos estudantes do Curso de Enfermagem de Vila Real, Região Norte, Trás-os-Montes e Alto Douro, município de Vila Real, Lordelo, em Portugal, comparando com outros cursos. Trata-se de um estudo descritivo, comparativo e transversal. Participaram na pesquisa 709 estudantes de sete cursos superiores, tendo-se procurado incluir atores que intervêm no processo de educação em saúde, aos quais foi aplicado um questionário de autopreenchimento, no período de outubro a dezembro de 2004 . A maioria dos participantes $(86,5 \%)$ considera que a educação em saúde tem valores a promover, sendo a 'responsabilidade' o valor mais indicado, por 19,2\% dos estudantes. O estudo demonstrou que é nos cursos de enfermagem que ocorre a maior evolução nos valores, existindo diferenças altamente significativas $(\mathrm{p} \leq 0,001)$, sendo a média do $4^{\circ}$ ano superior à do $1^{\circ}$. Esses resultados evidenciam a necessidade de, em tais cursos, se trabalhar os valores subjacentes ao processo, tão necessários no desempenho dessas profissões. Palavras-chave valores sociais; educação em saúde; capacitação; equidade.
Abstract The general purpose of this study was to examine the effect of training on the acquisition of values in health education among Vila Real Nursing School students, Northern Region, in Trás-os-Montes and in Alto Douro, in the municipality of Vila Real, Lordelo, Portugal, compared to those taking other courses. This is a descriptive, comparative, and transverse study. In total, 709 students from seven colleges took place in this research project, and an attempt was made to include authors intervening in the health education process, who replied to a self-completion questionnaire in the period ranging from October to December 2004. Most participants (86.5 percent) believe health education has values to promote, and 'responsibility' is the best value to 19.2 percent of the students. The study showed that it is in the nursing courses that most progress is made in terms of values, with highly significant differences ( $\mathrm{p} \leq 0.001)$. Additionally, it also revealed that the 4 th year students' average was higher than the 1st year's. These results are evidence of the need there is, in such courses, to work on the values underlying the process, which are so necessary for the performance of these professions. Keywords social values; health education; training; fairness. 


\section{Introdução}

No contexto atual, de ameaça de crises financeiras e preocupações daí emergentes, a promoção da saúde nunca foi mais oportuna, nem mais necessária do que hoje. Os documentos resultantes das Conferências Internacionais sobre Promoção da Saúde, organizadas pela Organização Mundial da Saúde, têm fornecido evidência científica e experiência acumulada sobre a importância desse processo como uma estratégia rentável e como um componente essencial dos sistemas de saúde (WHO, 2009). Por sua vez, a Ordem dos Enfermeiros de Portugal considera importante o exercício profissional no âmbito da promoção da saúde dos cidadãos, que está explicitamente incluída nas competências do enfermeiro de cuidados gerais (OE, 2003).

Sendo objetivos primordiais da prestação de cuidados de enfermagem promover a saúde e a qualidade de vida das pessoas e providenciar um ambiente humano e seguro, a educação em enfermagem deverá ter em conta esse direcionamento e preparar os estudantes para esse tipo de cuidados (Queirós, 2001). Essa preparação deverá envolver uma base teórica adequada, mas também dar atenção às atitudes, aos valores e às competências para que os futuros profissionais, chegados ao mercado de trabalho, possam prestar cuidados de qualidade e ser verdadeiros promotores da saúde da população e não concebam a educação em saúde (ES) apenas como um orientar e ensinar a prevenir doenças (Machado e Vieira, 2009).

Nesse sentido, um dos desafios que os docentes, em particular os formadores de enfermeiros, atualmente enfrentam é a educação para os valores dos seus estudantes. Mais do que identificar os valores dos estudantes, importa compreender o modo como eles os constroem e em que contextos (Marques, 2006). O estudo dessa problemática é importante, uma vez que permite identificar os valores assumidos pelos principais atores do processo de ES, bem como constituir um forte preditor do investimento que esses futuros profissionais irão fazer nas suas práticas e da sua eficácia.

Com vista a identificar os valores mais relevantes na área da promoção e educação em saúde (PES), procedemos, em estudos anteriores (Carvalho, 2007; Carvalho, A. e Carvalho, G., 2008; Carvalho, G. e Carvalho A., 2010), à análise de conteúdo de um conjunto de publicações nacionais e internacionais a fim de localizar referências a valores em PES. Para o efeito, analisámos 47 livros e artigos de revistas da especialidade internacionais, outros 19 de autores portugueses, bem como 30 documentos de organismos internacionais e 33 de organismos nacionais. Da análise desse vasto corpus documental, foi possível criar um conjunto de seis eixos de valores em PES: social-individual; salutogénico-patogénico; holístico-reducionístico; equi-dade-desigualdade; autonomia-dependência; democrático-autocrático, os quais constituem a matriz do presente estudo. 
Foi pois, neste quadro, que pretendemos investigar o efeito da formação universitária na aquisição e explicitação de valores em PES em estudantes de sete cursos de ensino superior, sendo quatro na área da saúde (um de medicina e três de enfermagem) e três da área das ciências sociais e humanas (educação de infância, ensino básico do $1^{\circ}$ ciclo e serviço social) tendo dado especial enfoque ao curso de enfermagem da Universidade de Trás-os-Montes e Alto Douro, em Vila Real por ser o curso em que estamos diretamente envolvidos. Para tal, traçamos como objetivo geral analisar o efeito da formação na aquisição de valores em ES dos estudantes do curso de enfermagem de Vila Real, comparando com outros cursos.

Neste sentido, formulámos os seguintes objetivos específicos: identificar os valores no âmbito da PES, que os estudantes detêm à entrada ( $1^{\circ}$ ano) e à saída da sua formação ( $4^{\circ}$ ano); comparar os sistemas de valores dos estudantes dos sete cursos participantes no estudo; verificar se a formação desenvolvida pelos cursos influencia os valores dos seus estudantes.

Sendo as pesquisas sobre valores em PES praticamente inexistentes, o presente estudo poderá contribuir para a produção de conhecimento inovador nesse âmbito e assim promover uma melhoria nos cursos de formação de enfermeiros.

\section{Métodos}

Este é um estudo descritivo, comparativo e transversal4 (Padilla e Haro, 2000; Parahoo, 2006), que pretende conhecer o sistema de valores relacionados com PES dos alunos do $1^{\circ}$ e do $4^{\circ}$ ano dos seguintes sete cursos universitários: enfermagem, da Universidade de Trás-os-Montes e Alto Douro, em Vila Real (E-VR); enfermagem, da Universidade do Minho, em Braga (E-BR); enfermagem, da Escola Superior de Enfermagem do Porto (E-PO); medicina, do Instituto de Ciências Biomédicas Abel Salazar da Universidade do Porto (MED); professores $1^{\circ}$ ciclo do ensino básico, da Universidade do Minho, em Braga (PEB); educação de infância, da Universidade do Minho, em Braga (EI); serviço social, da Universidade Católica Portuguesa, em Braga (SSO).

A população era constituída por 1.132 alunos e a amostra foi composta por todos os presentes no momento de recolha de dados e que voluntariamente preencheram os questionários, num total de 709 alunos, cerca de $62,6 \%$ do universo. Todos autorizaram a participação no estudo.

A escolha desta população obedeceu aos seguintes critérios: de especificidade, por incluir os estudantes de cursos relacionados com a PES, principal objeto deste estudo; de diversidade, pois fazem parte da pesquisa estudantes de cursos da área da saúde, da educação e de serviço social; e de abrangência, ao incluir os principais núcleos de formação, a nível do ensino superior, da região Norte de Portugal, quer do interior, quer do litoral. 
Os dados foram recolhidos por meio de um questionário de autopreenchimento, anónimo e confidencial, construído e devidamente validado (Pestana e Gageiro, 2005), o qual foi aplicado aos alunos do $1^{\circ}$ e do $4^{\circ}$ ano de cada um dos sete cursos participantes no estudo, no período de outubro a dezembro de 2004. Cumpriram-se todos os procedimentos éticos, com a obtenção de autorização oficial, por escrito, de cada instituição, além da realização de uma reunião prévia para informar acerca dos objetivos do estudo, garantindo a confidencialidade dos resultados e o anonimato dos respondentes. O órgão competente aprovou a realização do estudo. Não existem conflitos de interesses.

Na aplicação do questionário, verificaram-se duas situações distintas: nos cursos de enfermagem, os investigadores levaram, aplicaram e recolheram os questionários na data agendada para o efeito, enquanto nos cursos de MED, EI, PEB e SSO os questionários foram aplicados pelos próprios docentes e devolvidos aos investigadores.

A fim de avaliar a posição dos alunos dos diversos cursos face a cada um dos polos dos seis eixos de valores em PES, optámos por utilizar perguntas fechadas dicotómicas, para evitar que os respondentes assumissem posições neutras. Para cada eixo havia um conjunto de cinco questões. Para cada questão, os respondentes tiveram que escolher entre um polo ou o outro, que se situam em extremos opostos de cada eixo de valores: social-individual, salutogénico-patogénico, holístico-reducionístico, equidade-desigualdade, autonomia-dependência e democrático-autocrático.

Num primeiro passo, procedemos à codificação das variáveis, atribuindo o escore 1 a um polo (por exemplo, o polo individual no eixo social-individual) e o escore 5 ao outro polo (polo social). De seguida, por meio da janela Compute do programa SPSS, somaram-se os escores de cada grupo de cinco variáveis (questões), referente a cada eixo de valores, caso a caso, e dividiuse pelo número de variáveis. Obteve-se um escore final, que nos dá uma ideia do posicionamento do aluno, em relação a cada eixo de valores. O ponto de corte considerado foi o escore 3. Por último, tipificou-se o conjunto de alunos de cada curso, tendo em conta o escore obtido. Se menor que três, foi categorizado como pertencendo ao polo individual, no referido exemplo social-individual; no caso de ser maior ou igual a três, foi categorizado no polo social.

Para o tratamento dos dados recorremos à estatística descritiva e procedemos à realização de testes estatísticos de $\chi^{2}, t$ de Student, ANOVA, Mann-Whitney e Kruskal-Wallis, tendo-se utilizado o programa SPSS (Pestana e Gageiro, 2005). Considerámos existirem diferenças estatisticamente significativas quando $\mathrm{p}<0,05$. 


\section{Resultados}

Procedemos à caracterização dos indivíduos questionados quanto às variáveis independentes em estudo: género, classe etária e ano frequentado.

$\mathrm{O}$ 'sexo' feminino é largamente maioritário no total da amostra $(86,0 \%)$, bem como em cada curso; o de EI é exclusivamente frequentado por alunas $(100 \%)$ e o curso de MED é aquele onde o sexo masculino é mais representado $(23,6 \%)$, dentre os demais cursos.

A 'classe etária' predominante no $1^{\circ}$ ano é dos $17-19$ anos e no $4^{\circ}$ ano, dos 20-22 anos. A média de idades é de 20,3 anos, a moda é os 18,0 anos e o desvio padrão é de 2,29. Os três cursos de enfermagem (E-VR, E-BR e E-PO) são frequentados por alunos mais jovens do que os de MED e de PEB.

Quanto à 'promoção de valores em ES', quando questionados se "A educação em saúde tem valores a promover?", verificámos que $86,5 \%$ do total de alunos que constituem a amostra responderam "Sim", tendo sido os dos cursos de PEB e de E-BR os que mais advogaram tal opinião, respetivamente com $91,2 \%$ e 90,9\% dos alunos. A proporção de alunos dos vários cursos que consideram que a ES tem valores a promover varia significativamente $\left(\chi^{2}\right.$ : com $\left.\mathrm{p}<0,045\right)$, sendo os estudantes dos cursos de SSO $(78,2 \%)$ e de E-VR $(82,1 \%)$ os que estão menos conscientes da importância dos valores no âmbito da PES.

Existem diferenças estatísticas altamente significativas entre a proporção de estudantes do $1^{\circ}$ e do $4^{\circ}$ ano, que concordam que a ES tem valores a promover $\left(\chi^{2}\right.$ : com $\left.\mathrm{p}<0,001\right)$; no total da amostra havia $40,8 \%$ de estudantes do $1^{\circ}$ ano e $45,8 \%$ do $4^{\circ}$ ano que advogavam essa opinião.

Da resposta à questão aberta "A educação em saúde tem valores a promover? Se sim, indique esses valores", os alunos enunciaram oito valores diferentes, de forma isolada ou associada. No entanto, é de salientar que $39,3 \%$ dos alunos da amostra não conseguiram referir qualquer valor, 13,3 disseram que não veiculam valores e $6,2 \%$ não responderam a essa pergunta. Assim, apenas $41,2 \%$ explicitaram valores, tendo-se destacado o conceito 'responsabilidade' como o mais indicado de forma isolada $(19,2 \%$ dos alunos que indicaram valores a promover em ES, sendo seguido por 'respeito' e 'solidariedade', respetivamente, com 7,5\% e 6,5\%). Os alunos do $1^{\circ}$ e do $4^{\circ}$ ano indicaram um conjunto de valores muito semelhante. No entanto, a proporção de alunos do $4^{\circ}$ ano que citaram cada um dos valores é quase sempre mais expressiva que a dos alunos do $1^{\circ}$ ano (Tabela 1 ).

Em relação aos 'eixos de valores em ES', a partir das respostas ao grupo de questões relativas a cada um dos seis eixos de valores em PES previamente identificados - social-individual, salutogênico-patogénico, holístico-reducionístico, equidade-desigualdade, autonomia-dependência e democrático-autocrático -, procedeu-se ao posicionamento dos alunos de cada 
curso, em face dos respetivos polos. Apresenta-se a evolução ocorrida, entre as médias dos escores do $1^{\circ}$ e do $4^{\circ}$ ano, de todos os cursos avaliados, em cada conjunto de questões relativas aos eixos de valores e os resultados dos testes estatísticos que nos permitiram analisar as diferenças entre as médias dos dois anos, a fim de procurar aquilatar a influência da formação desenvolvida ao longo de cada curso.

Tabela 1

\begin{tabular}{|c|c|c|c|c|c|c|c|c|}
\hline $\begin{array}{ll}\text { Valores } & \text { Cursos }\end{array}$ & E-VR & E-BR & E-BR & MED & PEB & El & SSO & Total \\
\hline Responsabilidade & 1,0 & 3,1 & 7,2 & 3,1 & 1,7 & 2,1 & 1,0 & 19,2 \\
\hline Respeito & 0,34 & 2,1 & 2,1 & 0,68 & 0,34 & 1,0 & 1,0 & 7,5 \\
\hline Solidariedade & 0,68 & 1,7 & 2,1 & 0,0 & 0,68 & 0,68 & 0,68 & 6,5 \\
\hline Autonomia e responsabilidade & 1,4 & 0,68 & 3,4 & 0,34 & 0,0 & 0,34 & 0,0 & 6,2 \\
\hline Participação & 0,0 & 0,68 & 2,4 & 0,34 & 0,0 & 0,0 & 1,4 & 4,9 \\
\hline Cooperação & 0,0 & 0,34 & 1,4 & 0,0 & 0,34 & 0,0 & 0,34 & 2,4 \\
\hline Participação e responsabilidade & 0,0 & 0,34 & 1,0 & 0,34 & 0,34 & 0,34 & 0,0 & 2,4 \\
\hline Autonomia & 0,68 & 0,68 & 0,34 & 0,0 & 0,0 & 0,0 & 0,34 & 2,0 \\
\hline Cooperação, responsabilidade e & 0,0 & 0,68 & 0,0 & 0,0 & 0,68 & 0,34 & 0,34 & 2,0 \\
\hline solidariedade & & & & & & & & 2,0 \\
\hline Equidade & 0,0 & 0,0 & 0,34 & 0,68 & 0,0 & 0,0 & 1,0 & \\
\hline Combinações várias e outros valores & 8,2 & 7,2 & 12,3 & 4,8 & 3,4 & 5,1 & 3,8 & 44,9 \\
\hline Total dos valores explicitados & 12,3 & 17,5 & 32,6 & 10,3 & 7,5 & 9,9 & 9,9 & 100,0 \\
\hline
\end{tabular}

Fonte: Carvalho, Amâncio (2007).

*Apenas $41,1 \%$ dos respondentes explicitaram valores.

Nota: E-VR - enfermagem, da Universidade de Trás-os-Montes e Alto Douro, em Vila Real; E-BR - enfermagem, da Universidade do Minho, em Braga; E-PO - enfermagem, da Escola Superior de Enfermagem do Porto; MED - medicina, do Instituto de Ciências Biomédicas Abel Salazar da Universidade do Porto; PEB - professores $1{ }^{\circ}$ ciclo do ensino básico, da Universidade do Minho, em Braga; El - educação de infância, da Universidade do Minho, em Braga; SSO - serviço social, da Universidade Católica Portuguesa, em Braga.

Quanto à 'influência da formação desenvolvida nos diversos cursos', as médias dos escores obtidas pelos estudantes de cada curso (incluindo os do $1^{\circ}$ e os do $4^{\circ}$ ano), em cada um dos eixos, estão apresentadas na Tabela 2, assim como as médias dos escores por ano em cada curso na Tabela 3.

Em cada secção relativa aos eixos de valores, apresentam-se os resultados dos testes estatísticos, entre a média dos escores dos estudantes do $1^{\circ} \mathrm{e}$ do $4^{\circ}$ ano de cada curso. 
Tabela 2

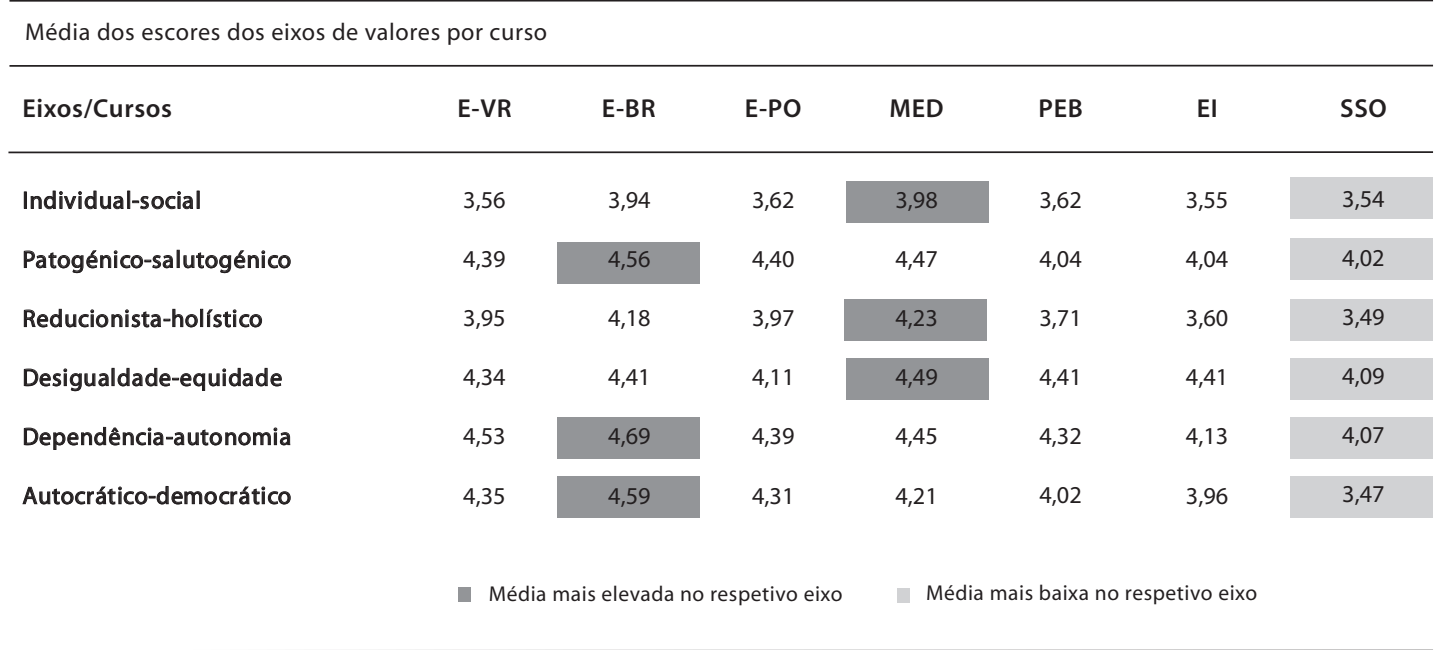

Fonte: Carvalho, Amâncio (2007).

Nota: E-VR - enfermagem, da Universidade de Trás-os-Montes e Alto Douro, em Vila Real; E-BR - enfermagem, da Universidade do Minho, em Braga; E-PO - enfermagem, da Escola Superior de Enfermagem do Porto; MED - medicina, do Instituto de Ciências Biomédicas Abel Salazar da Universidade do Porto; PEB - professores $1{ }^{\circ}$ ciclo do ensino básico, da Universidade do Minho, em Braga; El - educação de infância, da Universidade do Minho, em Braga; SSO - serviço social, da Universidade Católica Portuguesa, em Braga.

Tabela 3

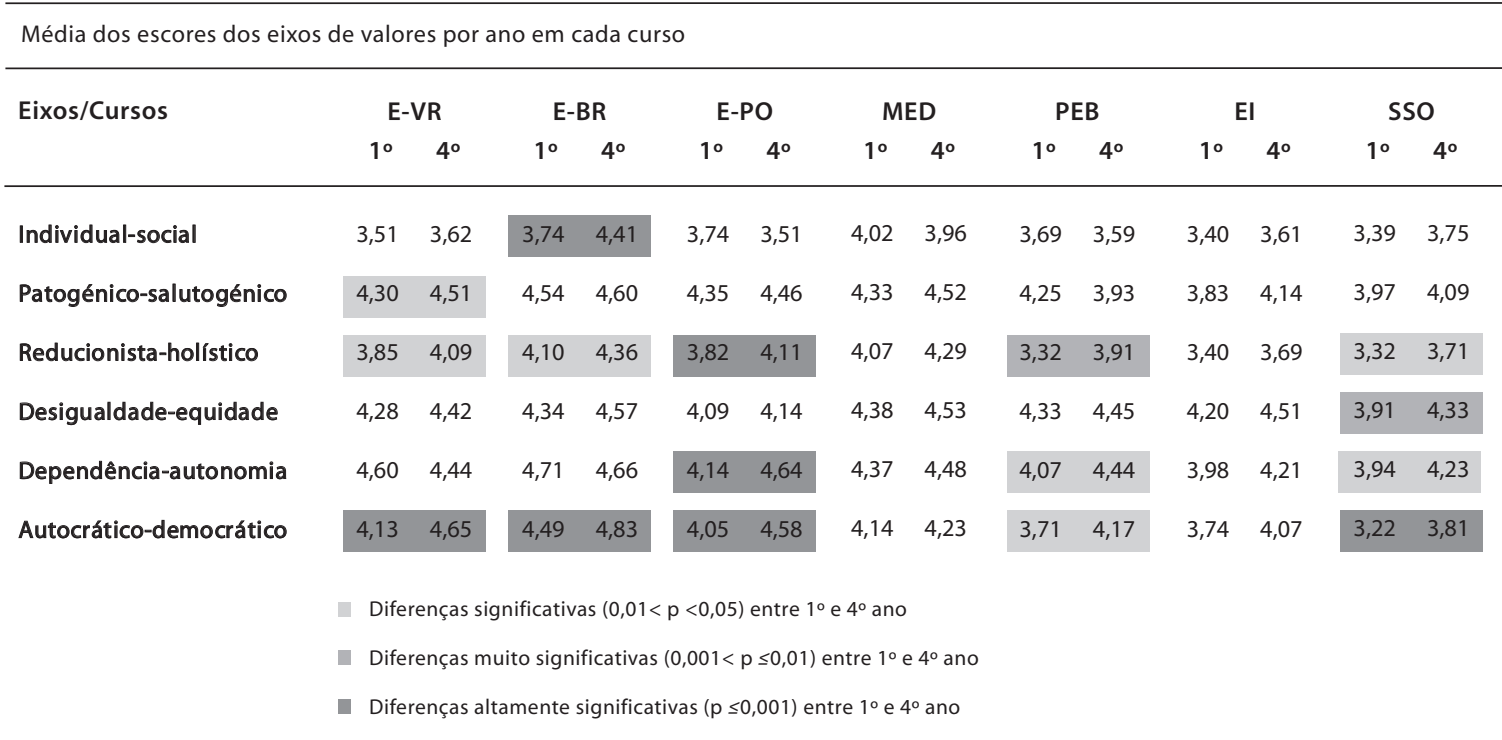

Fonte: Carvalho, Amâncio (2007).

Nota: E-VR - enfermagem, da Universidade de Trás-os-Montes e Alto Douro, em Vila Real; E-BR - enfermagem, da Universidade do Minho, em Braga; E-PO - enfermagem, da Escola Superior de Enfermagem do Porto; MED - medicina, do Instituto de Ciências Biomédicas Abel Salazar da Universidade do Porto; PEB - professores $1{ }^{\circ}$ ciclo do ensino básico, da Universidade do Minho, em Braga; El - educação de infância, da Universidade do Minho, em Braga; SSO - serviço social, da Universidade Católica Portuguesa, em Braga. 
No 'eixo social-individual', analisando as médias dos escores obtidos, por meio da valorização de perspetiva individual e de perspetiva social, verifica-se que existem diferenças altamente significativas (ANOVA: $p=0,000$ ) entre as médias dos escores dos sete cursos, especificamente entre os de E-BR e MED e os demais. O curso de MED foi aquele que obteve a média mais elevada $(3,98)$ e o de SSO a mais baixa $(3,54)$. O curso de E-VR situa-se entre estes extremos $(3,56)$.

Apenas no curso de E-BR existe diferença altamente significativa ( $t$ Student: $\mathrm{p}=0,000)$ entre a média dos escores da turma do $1^{\circ}$ ano $(3,74)$ e da turma do $4^{\circ}$ ano $(4,41)$, não havendo diferenças significativas entre os anos dos outros cursos.

No eixo salutogénico-patogénico, as diferenças existentes entre a média dos escores dos alunos, dos quatro cursos da área da saúde (E-VR, E-BR, E-PO e MED) e dos três cursos, que não são dessa área (PEB, EI e SSO), mostram-se altamente significativas (ANOVA: $p=0,000$ ). Os primeiros apresentam maiores médias do que os segundos (Tabela 2). A média mais elevada é a dos alunos de E-BR $(4,56)$ e a mais baixa pertence aos de SSO $(4,02)$. Os alunos do curso de E-VR $(4,39)$ posicionam-se na fronteira entre os cursos de saúde e os de educação e serviço social.

No curso de E-VR, e apenas neste, existem diferenças significativas ( $t$ Student: $\mathrm{p}=0,045$ ) entre as médias dos escores desse eixo dos alunos do $1^{\circ}$ e do $4^{\circ}$ ano; os do $4^{\circ}$ ano obtiveram uma média mais elevada $(4,51)$ do que os do $1^{\circ}$ ano $(4,30)$.

Tal como no eixo salutogénico-patogénico, também as diferenças existentes entre a média dos escores do eixo holístico-reducionista dos alunos dos quatro cursos da área da saúde (E-VR, E-BR, E-PO e MED) e dos outros três cursos (PEB, EI e SSO) são altamente significativas (ANOVA: p=0,000), com exceção do cruzamento entre o curso de E-VR e E-PO com PEB. A média dos escores mais elevada é a dos alunos do curso de $\operatorname{MED}(4,23)$ e a mais baixa continua a ser a dos alunos de SSO $(3,49)$.

Constataram-se diferenças estatísticas entre a média dos escores dos alunos do $1^{\circ}$ e do $4^{\circ}$ ano, sempre com superioridade do $4^{\circ}$ ano, que são altamente significativas no curso de E-PO ( $t$ Student: $\mathrm{p}=0,001)$, muito significativas no de PEB $(p=0,003)$ e significativas no de E-VR $(p=0,048)$, de E-BR $(p=0,025)$ e de SSO ( $t$ Student: $\mathrm{p}=0,028)$.

No eixo equidade-desigualdade, a média dos escores mais elevada foi encontrada nos alunos do curso de MED e a mais baixa foi, de novo, nos de SSO, registando-se diferenças estatísticas altamente significativas (ANOVA: $p=0,000)$ entre a média dos escores dos cursos, especificamente entre os de E-PO e SSO e os outros.

Quando se analisam as diferenças entre as médias dos escores do $1^{\circ}$ e do $4^{\circ}$ ano dos diversos cursos, apenas no de SSO se constataram diferenças muito significativas ( $t$ Student: $\mathrm{p}=0,004)$, sendo a média do $4^{\circ}$ ano mais elevada $(4,33)$ do que a do $1^{\circ}$ ano $(3,91)$. 
No eixo autonomia-dependência, constatou-se existirem diferenças altamente significativas (ANOVA: $p=0,000$ ) entre as médias dos escores. A média de valores mais expressiva é a dos alunos do curso de E-BR $(4,69)$ e a menos expressiva é a dos alunos de SSO $(4,07)$. Os alunos do curso de E-VR posicionam-se quase no topo dos cursos de saúde, mas não se encontram diferenças significativas entre os de saúde e os de educação e serviço social.

Analisando as diferenças existentes entre a média dos escores do $1^{\circ} \mathrm{e}$ do $4^{\circ}$ ano, ao nível de cada curso, constataram-se diferenças altamente significativas no de E-PO ( $t$ Student: $\mathrm{p}=0,000)$ e significativas nos de PEB $(\mathrm{p}=0,014)$ e SSO $(\mathrm{p}=0,016)$, sempre com supremacia da média do $4^{\circ}$ ano.

No eixo democrático-autocrático, existem diferenças altamente significativas (ANOVA: $p=0,000$ ) entre as médias dos escores dos sete cursos, especificamente entre a média do de SSO e de todos os outros. A média dos escores desse eixo mais elevada é de novo a dos alunos do curso de E-BR $(4,59)$, e a mais baixa, de SSO $(3,47)$.

Quando se analisam as diferenças existentes entre a média dos alunos do $1^{\circ}$ e do $4^{\circ}$ ano, de cada um dos cursos, verifica-se que são altamente significativas ( $t$ Student: $\mathrm{p}=0,000$ ) nos três cursos de enfermagem (Vila Real, Braga e Porto), bem como no de SSO, e são significativas no de PEB $(p=0,042)$, sendo a média sempre superior no $4^{\circ}$ ano.

\section{Discussão}

A bibliografia nacional e internacional relativa aos valores a serem desenvolvidos na formação de enfermeiros ou de outros profissionais, sejam eles de saúde ou de outras áreas, é muito escassa, pelo que optámos por focalizar a discussão do presente trabalho nos resultados obtidos no grupo de estudantes da E-VR, com quem trabalhamos diretamente, comparando com os dos outros cursos também de enfermagem (E-BR e E-PO), de medicina e de outros da área das ciências sociais e humanas (PEB, EI e SSO).

O facto de mais de $40 \%$ dos participantes não terem conseguido indicar qualquer valor em ES constitui um sinal claro da falta de preparação dos alunos para essa questão complexa. Para que os programas de ES não se limitem a iniciativas que visem apenas a informar a população, considerando os valores, os costumes e os modelos sociais, que conduzam a práticas educativas libertadoras, tal como afirmam Pinafo et al. (2011), o processo de ensino-aprendizagem terá de ter um olhar mais atento a essa questão. Dos valores que os alunos conseguiram expressar, o de 'responsabilidade' foi o que mais sobressaiu, aparecendo em primeiro lugar, seguido por 'respeito' e 'solidariedade'. Também um estudo realizado em Portugal (Oliveira, 1998), com uma amostra de 60 alunos do curso superior de enfermagem (bachare- 
lato) do $1^{\circ}$ ano (35 alunos) e do $3^{\circ}$ (25 alunos finalistas), na Região Autónoma da Madeira, hierarquizou os valores referidos pelos alunos pela ordem 'responsabilidade e tolerância', 'liberdade e igualdade', valores estes que são comuns aos do presente estudo.

O ligeiro, mas altamente significativo $\left(\chi^{2}: \operatorname{com} \mathrm{p}<0,001\right)$, aumento registado do $1^{\circ}$ ano para o $4^{\circ}$ ano (respetivamente, 40,8\% para $45,8 \%$ ) no conjunto dos sete cursos, mostra que o efeito da formação terá proporcionado um aumento na perceção sobre os valores em saúde. No entanto, atenção deve ser dada também à influência da idade/maturação dos jovens entre o $1^{\circ}$ ano (17-19 anos) e o $4^{\circ}$ ano (20-22 anos) na consciencialização dos sistemas de valores, particularmente em saúde. Na verdade, neste estudo, as variáveis 'ano de escolaridade' e 'idade' não são dissociáveis. Por essa razão, comparámos essa evolução nos sete cursos, sabendo que quatro são da área da saúde e três de ciências sociais e humanas, com vista a verificar qual o efeito da formação.

O curso de SSO foi aquele que, para todos os eixos de valores, teve os escores mais baixos, significando, por isso, serem mais sensíveis às questões dos foros individual, patogénico, reducionista, desigualdade, dependência e autocracia. Pelo contrário, os alunos de MED são os mais próximos dos polos social, holístico e equidade, e os do curso de E-BR dos polos salutogénico, autonomia e democracia. Esses resultados indicam que as conceções sobre os valores em ES são significativamente distintas entre os estudantes dos cursos da área da saúde e os da área das ciências sociais e humanas.

O presente estudo mostrou claramente que é nos cursos da área da saúde que ocorre uma maior evolução dos valores em ES (altamente significativa: $\mathrm{p} \leq 0,001)$, nomeadamente no curso de E-PO (nos eixos holístico-reducionista, autonomia-dependência e democrático-autocrático), de E-BR (social-individual e democrático-autocrático) e de E-VR (democrático-autocrático). Dos cursos de ciências sociais e humanas, apenas o de SSO apresentou uma alteração altamente significativa, também no eixo democrático-autocrático. Na verdade, esse eixo foi o único que teve um aumento altamente significativo em mais do que um curso: todos os de enfermagem e o de serviço social.

Esses resultados vão ao encontro de conclusões de outras pesquisas, que afirmam que estudar durante quatro anos numa escola de enfermagem conduz à diferença de valores, em que o ano de matrícula é um fator influente em alguns valores (Shinyashiki et al., 2006).

Dois dos eixos não tiveram aumento altamente significativo $(\mathrm{p}<0,001)$, entre o $1^{\circ}$ e o $4^{\circ}$ ano (ver Tabela 3), tendo apresentado apenas aumento muito significativo $(p<0,01)$. São eles o eixo salutogénico-patogénico no curso de E-VR e equidade-desigualdade, no de SSO. Esses resultados são muito interessantes, pois demonstram que o curso de enfermagem enfatizou 
os valores da sua área específica de saúde (pertencentes ao eixo salutogénico-patogénico), enquanto que o de serviço social na sua área específica de questões sociais (equidade-desigualdade).

Dos sete cursos, destacam-se dois, o de MED e EI que não registaram diferenças significativas ( $p>0,05$ em todos os eixos) entre o $1^{\circ}$ e o $4^{\circ}$ ano, cujas causas, no caso do de MED, se pode dever ao fato de os escores no $1^{\circ}$ ano já serem bastante mais elevados do que nos outros cursos e, por isso, o efeito da formação não terá sido tão sensível como nos outros cursos.

No que diz respeito ao curso de E-VR em que estamos diretamente envolvidos, podemos verificar que onde se deu uma maior evolução de valores entre o $1^{\circ}$ e o $4^{\circ}$ ano foi no eixo democrático-autocrático $(\mathrm{p}<0,001)$, seguido pelo salutogénico-patogénico e holístico-reducionista $(\mathrm{p}<0.05)$. Embora tenha havido aumento dos escores nos eixos social-individual, equidade-desigualdade e autonomia-dependência, não foram estatisticamente significativos ( $p>0,05)$, indicando que estes três últimos eixos deverão merecer mais atenção no processo de formação do curso de E-VR.

No seu todo, e em termos de conclusão, os resultados do presente estudo mostram que deverá ser dada maior atenção: à tendência social em todos os cursos, exceto no de E-BR; à tendência salutogénica em todos os cursos, menos no de E-VR; à tendência holística nos cursos de EI e MED; à tendência da equidade em todos os cursos, à exceção do SSO; à tendência da autonomia em todos os cursos, exceto E-PO, PEB e SSO; à democracia nos cursos de MED e de EI. Os cursos de saúde parecem, assim, desenvolver mais a perspetiva holística nos seus alunos, indo ao encontro do preconizado no ensino de enfermagem: um desenvolvimento holístico (Scherer, Z., Scherer, E. e Carvalho, 2006).

\section{Considerações finais}

Tendo em conta os objetivos traçados à partida e os resultados obtidos no presente estudo, conclui-se que os estudantes detêm à entrada ( $1^{\circ}$ ano) um conjunto de valores em PES muito semelhante ao detido pelos estudantes à saída da formação ( $4^{\circ}$ ano) dos diversos cursos incluídos no estudo, do qual fazem parte valores como responsabilidade, respeito, solidariedade, participação, autonomia, equidade e cooperação. No entanto, a proporção de estudantes do $4^{\circ}$ ano a identificar esses valores é quase sempre superior à do $1^{\circ}$ ano, demonstrando que a formação desenvolvida nos cursos aumenta a perceção sobre os mesmos.

Também podemos concluir que as conceções sobre os valores em PES são distintas entre os cursos da área da saúde e os da área das ciências sociais e humanas. Nos de enfermagem, ocorre uma maior evolução dos valores. Esses 
cursos enfatizam os valores social, holístico, autonomia e democrático. O de E-VR, por sua vez, dá relevo ao valor salutogénico, enquanto o de SSO se direciona para a equidade. Além disso, a formação desenvolvida na maioria dos cursos (exceto MED e EI) parece influenciar os valores dos seus estudantes.

O estudo é pertinente, pois salienta a baixa consciencialização que os alunos dos cursos nele incluídos têm sobre os valores em ES, a necessidade de trabalhar esses conteúdos atitudinais e de apontar os valores aos quais deve ser dada maior atenção em cada um dos cursos. A formação em enfermagem, especificamente, não pode ser alheia à questão dos valores.

\section{Colaboradores}

Amâncio Carvalho e Graça Carvalho trabalharam em conjunto no desenvolvimento desta pesquisa e em todas as etapas de produção do manuscrito, que também contou com a participação de Vítor Rodrigues em sua elaboração.

\section{Nota do Editor}

Trabalho, Educação e Saúde manteve a redação original deste artigo no português corrente em Portugal.

\section{Notas}

1 Professor Adjunto do Departamento de Enfermagem de Saúde Mental e Comunitária (DESMC), da Escola Superior de Enfermagem de Vila Real, da Universidade de Trás-os-Montes e Alto Douro (ESEVR-UTAD), Vila Real, Portugal. Doutorado em Estudos da Criança (Ciências da Educação), com Especialização em Saúde Infantil, pela Universidade do Minho (UM), Braga, Portugal.<amancioc@utad.pt>

Correspondência: Rua das Pintas, s/n, Vila Marim, 5000 - 773 Vila Real, Portugal. 
2 Professora Catedrática com agregação, Doutoramento em Biologia, Departamento de Estudos Integrados de Literacia, Didáctica e Supervisão, Instituto de Educação da Universidade do Minho, Centro de Investigação em Estudos da Criança (CIEC), Braga, Portugal. <graça@ie.uminho.pt>

3 Professor Coordenador com Agregação da Escola Superior de Enfermagem/Centro de Investigação em Desporto, Saúde e Desenvolvimento Humano da Universidade de Trás-os-Montes e Alto Douro, Vila Real, Portugal. Doutor em Ciências Biomédicas pela Universidade do Porto, Portugal. <vmcpr@utad.pt>

4 Esta pesquisa teve o suporte financeiro do Centro de Investigação em Formação de Profissionais de Educação da Criança/Centro de Investigação em Estudos da Criança (CIFPEC/CIEC), unidade de investigação 644/317 da FCT, e resultou de uma tese de doutorado na Universidade do Minho do autor Amâncio Carvalho, tendo sido aprovada pelos órgãos competentes das instituições envolvidas na coleta de dados.

\section{Referências}

CARVAlHO, Amâncio A. Promoção da saúde: concepções, valores e práticas de estudantes de enfermagem e de outros cursos do ensino superior, 2007. Tese (Doutorado em Estudos da Criança - Ciências da Educação) - Instituto de Estudos da Criança, Universidade do Minho, Braga, 2007.

CARVALHO, Amâncio A.; CARVALHO, Graça S. Eixos de valores em promoção da saúde e educação para a saúde. In: PEREIRA, Beatriz; CARVALHO, Graça (Eds.). Actividade física, saúde e lazer: modelos de análise e intervenção. Lisboa: Lidel, 2008. p. 195-205.

CARVALHO, Graça S.; CARVALHO, Amâncio A. Axes of Values in Health Education: Identification, Characterization and Application to Students of Seven University Cour-ses. In: IOSTE SYMPOSIUM (INTERNATIONAL ORGANIZATION FOR SCIENCE AND TECHNOLOGY EDUCATION) - SOCIO-CULTURAL AND HUMAN VALUES IN SCIENCE AND TECHNOLOGY EDUCATION, 14, 2010. Bled, Slovenia, 2010. CD-ROM.

MACHADO, Maria F.; VIEIRA, Neiva F. Health Education: The Family Health
Teams' Perspective and Clients Participation. Revista Latino-Americana de Enfermagem, Ribeirão Preto, v. 17, n. 7, p. 174179, 2009.

MARQUES, Paula A. Os valores dos jovens e a educação para os valores: o caso particular da formação moral dos estudantes de enfermagem. Revista Portuguesa de Enfermagem, Amadora, n. 7, p. 13-15, 2006.

OLIVEIRA, Maria L. Escola: encontro de pessoas. Que valores? Perspectiva dos alunos de curso superior de enfermagem. Servir, Lisboa, v. 46, n. 5, p. 248-258, 1998.

ORDEM DOS ENFERMEIROS (OE). Conselho de Enfermagem. Competências do enfermeiro de cuidados gerais, 2003. Disponível em: $<$ www.ordemenfermeiros.pt/publicacoes/ Documents/competenciasEnfCG.pdf $>$. Acesso em: 21 fev. 2011.

GARCIA PADILLA, Francisca; GONZÁLEZ HARO, María. Estudios descriptivos. In: OSUNA, António Frías (Org.) Salud Pública y Educación para la Salud. Barcelona: Masson, 2000. p. 129-140. 
PARAHOO, Kader. Nursing Research: Principles, Process and Issues. 2. ed. London: Palgrave Macmillan, 2006.

PESTANA, Maria H.; GAGEIRO, João N Análise de dados para ciências sociais: a complementaridade do SPSS. 4. ed. Lisboa: Sílabo, 2005.

PINAFO, Elisangela et al. Relação entre concepções e práticas de educação em saúde na visão de uma equipe de saúde da família. Trabalho, Educação e Saúde, Rio de Janeiro, v. 9, n. 2, p. 201-221, jul.-out. 2011.

QUEIRÓS, Ana A. Ética e enfermagem. Coimbra: Quarteto, 2001.
SCHERER, Zeyne A.; SCHERER, Edson A.; CARVALHO, Ana M. Reflections on Nursing Teaching and Students' First Contact with the Profession. Revista Latino-Americana de Enfermagem, Ribeirão Preto, v. 14, n. 2, p. 285-291, 2006.

SHINYASHIKI, Gilberto T. et al. Professional Socialization: Students Becoming Nurses. Revista Latino-Americana de Enfermagem, Ribeirão Preto, v. 14, n. 4, p. 601-607, 2006. WORLD HEALTH ORGANIZATION (WHO). Overview: 7th Global Conference on Health Promotion. The Urgency of Health Promotion, 2009. Disponível em: <www.who.int/ healthpromotion/conferences/7gchp/overview/ en/index.html> . Acesso em: 21 fev. 2011.

Recebido em 12/03/2012

Aprovado em 09/07/2012 